\title{
Urgences
}

\section{Un comte russe de par chez nous (extrait)}

\section{Pauline Cadieux}

Numéro 9, 1er trimestre 1984

URI : https://id.erudit.org/iderudit/025143ar

DOI : https://doi.org/10.7202/025143ar

Aller au sommaire du numéro

Éditeur(s)

Urgences

ISSN

0226-9554 (imprimé)

1927-3924 (numérique)

Découvrir la revue

Citer ce document

Cadieux, P. (1984). Un comte russe de par chez nous (extrait). Urgences, (9), 83-90. https://doi.org/10.7202/025143ar d'utilisation que vous pouvez consulter en ligne.

https://apropos.erudit.org/fr/usagers/politique-dutilisation/ 
PAULINE CADIEUX

\title{
UN COMTE RUSSE DE PAR CHEZ NOUS
}

\author{
(extrait)
}


Dès ses années de collège puis de Petit séminaire, Antoine-Auguste Levallier avait informé ses confrères de tous les détails de sa généalogie, persuadé que son lointain aïeul avait porté une particule devant son nom et ne l'avait laissé tomber que pour être mieux accepté parmi les petites gens formant alors la colonie. Et il ne rêvait que de la reprendre, ce qui ne lui facilita pas les choses au point de vue popularité.

Les jeunes de cette époque étaient francophiles à souhait. On ne parlait que des grands poètes et des grands écrivains français. On en était encore chez les étudiants à Musset, de Vigny, Lamartine, Hugo. L'abbé Groulx commençait ses cours d'histoire. Depuis 1917 s'étaient fondées à Montréal, deux revues largement distribuées dans nos collèges classiques, I'Almanach de la langue française et l'Action française.

Mais si tout concourait à ce que les petits québécois se rapprochent de plus en plus de leurs origines françaises, personne chez les adolescents et les étudiants ne se pâmait d'admiration devant un des leurs prétendant descendre en ligne directe, ou indirecte, d'un noble français, ou se disant tel.

Le premier du nom Levallier était venu directement de France, sur la pointe des pieds: son passage avait été payé par le roi lui-même, qui lui avait aussi octroyé, non pas une seigneurie, mais un fort bon lopin de terre et l'avait nanti d'une rente qui ne s'éteignit qu'à sa mort.

Beau garçon, âgé de dix-neuf ans, sans métier, de blanches mains et de beaux habits, il eut la chance qu'aucun Iroquois ne lui enleva la chevelure ou la vie. Une accorte jeune fille lui confia sa destinée et le foyer se peupla de quatre garçons et d'autant de filles, lesquelles à leur tour, prirent maris. Mais il arriva que seul I'un des garçons eut une descendance, la guerre d'escarmouches n'ayant pas épargné les fils comme elle avait épargné le père.

En hommage au roi, François Levallier, celui de France, avait fait donner à son premier né le prénom d'Auguste. Ce fut celui qui fit souche. A son tour Auguste crut devoir donner son 
prénom à son fils aîné, ça se faisait beaucoup dans la colonie, mais il y ajouta un autre patronyme et ce fut celui de François le grand'père: ce qui donna François-Auguste. Et ainsi s'établit la coutume. II y eut des Charles-Auguste, Jacques-Auguste, Simon-Auguste, etc, pour dix générations de Levallier canadiens.

Le véritable but de la venue de François Levallier, à la fin du dix-septième siècle, ne fut jamais éclairci à fond. Peut-être ne le connaissait-il pas vraiment lui-même. Ca arrivait à ce moment-là. Il avait parlé vaguement à ses enfants, et plus tard à ses petits-enfants, et ça s'était transmis de père en fils, de sa vie antérieure, en France, qui semblait s'être déroulée dans une ambiance, sinon de grand luxe, du moins de confort et d'aisance. D'ailleurs, n'avait-il pas une rente du roi? Quelque minime qu'elle fût, c'était une distinction.

En Nouvelle-France, à cette époque, on avait d'autres soucis que de chercher qui était qui et pourquoi il se trouvait ici plutôt qu'ailleurs. Et lorsqu'à la troisième ou quatrième génération on eut le loisir de se poser des questions plus précises, il était trop tard pour élucider toute I'affaire, l'aïeul ayant trépassé sans fournir plus d'éclaircissements que ceux qu'il avait ébauchés.

De père en fils, on se transmit l'interrogation: François Levallier était-il le fils naturel d'un noble quelconque qui ne pouvait ou ne voulait le reconnaître? Pourtant, ça se faisait couramment en France à ce moment-là! Pourquoi le Roi luimême avait-il tenu à assurer sa subsistance? On ne le sut jamais.

Les Levallier avaient prospéré sur la terre octroyée à l'aïeul et, au début du vingtième siècle, lorsqu'AntoineAuguste parut en ce monde, son père, Dieudonné-Auguste, lui-même benjamin d'une famille qui comptait déjà sept filles, fit chanter une messe d'action de graces: on avait craint la fin de la dynastie! Comme s'il y avait eu un droit d'aînesse!

Dieudonné-Auguste avait hérité de son père, Séraphin- 
Auguste, un bien magnifique, de la belle terre franche, planche, à perte de vue, qui poussait bien. Déjà nanti de deux filles, il avait requis son gendre, le mari de sa fille aînée, Augustine, qui s'était mariée à seize ans, de venir le seconder.

On échafauda bien des rêves à la naissance d'AntoineAuguste: le père avait des projets pour son fils: on n'avait pas encore eu de prêtres dans la famille... ni de religieuses, d'ailleurs.

Dieudonné lui-même était plutôt déçu de la monotonie du train-train de vie que I'on menait. II était allé au séminaire pendant quatre ans et il avait épousé une fille de la paroisse, qui, elle, avait été institutrice.

On lisait beaucoup chez ce couple durant les longs mois d'automne et d'hiver et l'on s'intéressait à toutes les nouvelles initiatives que rapportaient les gazettes que I'on recevait avec un peu de retard. Ainsi, dès le tout début du siècle, après la naissance de l'héritier, on s'était pris d'enthousiasme pour un projet mis de l'avant par des promoteurs montréalais, voulant construire un chemin de fer électrique sous le nom de Chemin de fer électrique de Terrebone, pour le transport des passagers seulement, mû par l'électricité, pour l'avantage du public, passant par toutes ou chacune des paroisses, villages, villes ou cités des comtés d'Hochelaga, Jacques-Cartier, Laval, Argentueil, Wright, Labelle, Terrebonne, L'Assomption et Montcalm, avec des voies passant par toutes les rues des dits endroits. C'était trop beau et ça ne se réalisa jamais.

Cependant, ces messieurs, de par leur loi qui avait été sanctionnée le deux juin mil neuf cent quatre, pouvaient construire, équiper, entretenir et exploiter des lignes de télégraphe et de téléphone sur toute la longueur de son chemin de fer et de ses embranchements, pour son usage, et elle pouvait vendre le surplus d'électricité qu'elle produirait pour être utilisé comme calorique, luminaire ou force motrice, dans les limites des comtés qu'ils traverseraient.

Le capital-actions de la compagnie étant de deux millions 
de dollars, somme énorme pour l'époque, les directeurs, Louis Payette, entrepreneur, Héliodore Fortier, marchand, Richard Wilson-Smith, courtier, H.E. Ekers, brasseur, tous de la cité de Montréal, et John E.E. Dickson, gérant d'assurance et Alexander R. Johnson, avocat, de la ville de Westmount, tentaient d'intéresser le public à devenir actionnaire dans ce projet.

Dieudonné ne savait pas trop s'il devait souscrire des parts dans cet organisme, mais Marie-Anne, sa femme, était absolument emballée: on pourrait avoir l'électricité dans la maison et les bâtiments, on pourrait voyager et on ferait partie d'une très grosse compagnie!

II n'existait aucun courtier dans le patelin ni non plus dans la ville voisine et le notaire, consulté, ne fut d'aucun secours: ce n'était pas un progressiste! Les Levallier ne devinrent donc pas partenaires du chemin de fer électrique de Terrebonne, lequel, bien qu'il fut constitué en corporation, n'opéra jamais.

On avait aussi été frappé par la vulgarisation que faisaient les savants à cette époque, que certains mammifères étaient devenus amphibies au cours des ans, fréquentant dorénavant surtout les mers. II n'y avait pas de mer ni d'océan à la ronde, mais une rivière passait au bout de la terre et DieudonnéAuguste et Marie-Anne avaient longtemps supputé les gains qu'ils pourraient réaliser si certains gorets s'habituaient à vivre dans l'eau: ce serait alors de la "chair maigre" que l'on pourrait manger en tout temps surtout pendant le carême, comme on le faisait pour la morue séchée! Personne n'aimait la morue séchée, mais par quoi la remplacer?

Pas mal de générations de gorets furent perdues dans cette expérience: dans leur enclos des bords de la rivière, certains s'étaient noyés, d'autres étaient devenus perclus, des truies avaient mangé leurs petits, sans doute pour leur éviter un sort plus pénible et les voisins n'avaient pas cessé leurs railleries. Déçu de ne pouvoir faire une exploitation agricole différente de celles de ses voisins, Dieudonné-Auguste n'aimait pas qu'on le raillât trop souvent à la sortie de la messe. Comment échapper à tout cela? Ce fut en revenant de la messe 
au village que Marie-Anne trouva la solution.

Elle avait remarqué, sur la rue principale qu'une maison était à vendre: passablement grande, avec un bon terrain autour. On arrivait tous deux à la quarantaine, ce serait idéal pour s'y habituer au repos et pour y emménager avec le reste des enfants qui pourraient fréquenter les écoles du villages. Gilbert, le mari d'Augustine, garderait la terre ancestrale, qu'il exploitait d'ailleurs aussi bien que s'il eut été un vrai Levallier.

Dieudonné-Auguste était bien d'accord mais avec quel argent vivrait-on? On pourrait peut-être obtenir les victuailles de Gilbert sur la production agricole, mais il fallait plus que cela. On résolut d'y réfléchir, ce qui prit encore quelques semaines.

Les récoltes engrangées, Dieudonné-Auguste et son gendre Gilbert étant allés faire quelques emplettes au magasin général où ils s'attardèrent, rencontrèrent, comme tout un chacun, un certain monsieur Saint-Onge, qui se disait inspecteur pour une compagnie d'assurance-vie et qui cherchait un représentant pour la région. C'était une occupation nouvelle dont on ne connaissait pas très bien les aléas et les possibilités et le pauvre inspecteur, tout "monsieur" qu'il paraissait être, ne recruta personne.

De retour à la maison, les hommes reprirent la question au dîner devant Marie-Anne qui n'en crut pas ses oreilles: c'était cela, la solution au problème du gagne-pain si on s'installait au village! Personne dans la région n'avait d'assurances, alors ce serait autant de clients en perspective. Dieudonné-Auguste commença de regretter d'avoir tant parlé. Le farniente lui souriait assez mais la discussion avec ses concitoyens le glaçait. Ah! si les femmes avaient été éligibles à une telle chance, c'est Marie-Anne qui aurait sauté dessus!

Marie-Anne mit un bon gros mois à convaincre son mari de rentrer en communication avec monsieur Saint-Onge. On verrait bien ce qu'il en était. Monsieur Saint-Onge connaissait son affaire et c'est un Dieudonné-Auguste pas trop convaincu mais prêt à tenter l'aventure qui consentit à signer le contrat d'engagement qu'on lui tendit. Marie-Anne jubilait. Enfin, ter- 
miné le gros ouvrage, elle pourrait maintenant "faire sa dame" ce dont elle avait toujours rêvé depuis sa lointaine expérience d'institutrice.

Le vingtième siècle avait déjà quelques années lorsqu'Antoine-Auguste devint villageois, son père ayant troqué son statut de cultivateur pour celui d'homme d'affaires. Dieudonné mit quelque temps à entrer dans la peau de son nouveau personnage. Sa femme avait transformé toute la maison qu'il n'avait pas encore vendu trois polices d'assurances. On n'irait pas loin comme ça.

C'est que Dieudonné ne s'était jamais rendu compte qu'il était timide! Oh! avec les femmes, bien sûr, dans les soirées, à la campagne, il n'avait pas eu son pareil pour les frôler et pour peloter les créatures qui lui tombaient entre les pattes lors des "Paul-Jones": c'était bien connu et, mine de rien, pour ne pas offenser Marie-Anne, si serviable et si gentille, on s'arrangeait toujours pour le fuir; tout le monde n'y arrivait pas! Dans le monde des assurances, c'était avec le mari qu'il fallait transiger et les "mains volantes" n'étaient pas alors d'un grand secours.

De surplus, endimanché tous les jours, Dieudonné se sentait gêné aux entournures. Mais enfin, poussé dans le dos par sa femme et aguiché par certaines villageoises qui n'avaient pas froid aux yeux, il s'enhardit et, après deux ans, si les cultivateurs ne lui avaient pas acheté une seule police, il en avait vendu une dans presque toutes les maisons du bourg. L'inspecteur lui proposa alors de s'installer dans une petite ville, à une vingtaine de milles de là, à Saint Clin-Clin, pour de l'assurance générale.

Marie-Anne qui avait pris goût, le dimanche, de se rendre aux réunions des archi-confréries, qui participait aux tombolas et bazars de l'église et des bonnes oeuvres, qui prenait part aux décisions des dames patronnesses, n'était pas mécontente d'élargir son champ d'action.

Pratiquement sans effort, on vendit la maison avec un 
assez bon profit et l'on put se réinstaller dans une autre grande maison. Ce ne fut pas toute de suite que Dieudonné-Auguste se rendit compte des avantages immenses de la galerie qui longeait la rue principale, sur le devant de la maison, et qui empiétait même sur le trottoir d'un pouce ou deux!

II installa sa berceuse en face de la fenêtre d'une grande pièce du rez-de-chaussée que Marie-Anne avait baptisée du titre pompeux de "bureau", tandis que l'autre fenêtre de la façade éclairait une immense pièce dont on fit un salon. Sur un côté se trouvait la maison du notaire et de l'autre côté se trouvait l'office du docteur. Qu'est-ce qu'on pouvait demander de mieux comme environnement? 